\title{
BRCA1/2 and clinical outcome in a monoinstitutional cohort of women with hereditary breast cancer
}

\author{
DOMENICO SAMBIASI, ROSSANA LAMBO, BRUNELLA PILATO, STEFANIA TOMMASI, GIUSEPPE TROJANO, \\ ANILA KARDHASHI, MARIA DIGENNARO, VITO TROJANO, GIANNI SIMONE and ANGELO PARADISO
}

National Cancer Research Centre ‘Giovanni Paolo II’, I-70124 Bari, Italy

Received November 15, 2012; Accepted December 17, 2012

DOI: 10.3892/or.2013.2802

\begin{abstract}
The clinical outcome of BRCA mutation carriers and non-carriers still remains a topic of discussion. In order to interpret controversial data, in the present study, we analyzed a large consecutive monoinstitutional series of breast cancer patients and relatives with familial features carrying or not carrying BRCA mutations. The intense research in recent years regarding the clinical genetics of patients with breast or ovarian cancer and their relatives has allowed the organization of a unique database comprising anamnestic, clinical, pathological and molecular data. Families with two or more cases of breast cancer under the age of 50 years, or with three cases at any age, were identified. From June, 2003 to June, 2010 , a total of 202 patients (136 probands +66 relatives) from 45 families were included in the analysis. A total of 136 (49 carrier and 87 non-carrier) cases had a cancer diagnosis at the time of their genetic testing. Twenty and 24 events were observed in the carrier and control group, respectively. The 10-year disease-free suvival rate was $57 \%$ for patients in the control group compared with $50 \%$ for patients carrying a BRCA mutation ( $\mathrm{P}=0.15$ by $\log$-rank test). Finally, 66 (32 genetic and 34 control) cases were unaffected at the time of molecular analysis, and 6 new cases of cancer were observed in the carriers, while no new cases were detected in the control cohort. Thus, at age $50,40 \%$ of carriers had a high risk of disease $(\mathrm{P}=0.0069$ by $\log$-rank test). Our data support the hypothesis that the presence of BRCA mutations does not alter the clinical outcome for hereditary breast cancer patients. Conversely, BRCA mutations are proven to be crucial for prediction of risk in healthy relatives from carrier families.
\end{abstract}

\section{Introduction}

Mutations in autosomal dominant genes are responsible for $\sim 5-10 \%$ of all breast cancers and $\sim 7-10 \%$ of all ovarian

Correspondence to: Dr Angelo Paradiso, Scientific Division, National Cancer Research Centre, IRCCS 'Giovanni Paolo II', O. Flacco 65, I-70124 Bari, Italy

E-mail: a.paradiso@oncologico.bari.it

Key words: BRCA1, BRCA2, follow-up, outcome cancers (1). Specifically, inherited mutations in the BRCA1 and BRCA2 genes predispose women to both breast and ovarian cancers, often at young ages. Women who carry these BRCA1 or BRCA 2 mutations have an estimated lifetime risk of $60-85 \%$ and of $26-54 \%$ for breast and ovarian cancer, respectively (2-5). An increased cancer risk is also recognized in men with BRCA2 mutations, conferring a $6 \%$ lifetime risk of breast cancer and a 3- to 7-fold increased risk of developing prostate cancer $(6,7)$.

Moreover, having one or more close relatives with breast cancer is an important and well-established risk factor for that disease, with the magnitude of risk varying depending on the number of affected relatives and the ages at which the relatives were diagnosed (8).

Several authors have looked at BRCA alterations not only as a biomarker of risk for breast cancer but also as a marker of clinical pathological aggressiveness (9); however, previous studies have provided results which are frequently controversial, with some studies demonstrating that BRCA1 mutation carriers develop tumors with a higher proliferative capability and low estrogen receptor levels $(10,11)$ while others have reported the lack of a difference in histological tumor features among BRCA2-positive familial and sporadic cases (12).

A large population study (13) found that the 10-year survival rate did not differ for BRCA $1 / 2$ mutation carriers and non-carriers, leading to the general opinion that woment with a BRCA1 or BRCA2 gene mutation diagnosed with breast cancer have similar survival with respect to that of non-carriers.

Moreover, breast cancer-specific mortality rates have been found to be similar for BRCA mutation carriers and noncarriers in a Jewish population (13). Analogously, in Sardinia, whose population is genetically homogeneous, investigators did not find any significant difference in outcome between patients carrying BRCA2 mutations and those negative for $B R C A 2$ mutations (most prevalent BRCA sequence variations), as well as no difference in survival among familial and sporadic BRCA2 mutatation cases (14).

Following a diagnosis of breast cancer in one breast, a woman with a BRCA1 or BRCA 2 mutation experiences a high risk for contralateral breast cancer (CBC). The 10-year risk of $\mathrm{CBC}$ has been estimated to be between 20 and $30 \%$. Factors that predict the risk of $\mathrm{CBC}$ include early age at diagnosis of the first breast cancer and a family history of early-onset breast cancers in first-degree relatives (15-18). 
Notably, these data were frequently obtained from a nonconsecutive series of patients, pooling molecular information from a multitude of laboratories, with patients receiving primary surgery and histopathological diagnosis from different professionals, undergoing treatment with extremely different systemic approaches and being followed up using various modalities. This was carried out in spite of the fact that each of these factors has been repeatedly and clearly demonstrated to be able to deeply influence the outcome of breast cancer women (19-21).

The Outpatient Clinic for Hereditary Breast/Ovarian Cancer has been active at the National Cancer Research Centre (NCRC), Bari, Italy since 2003 (22). It includes a dedicated genetics laboratory with extensive experience in BRCA analysis (24) and is actively involved in national and international QC programs (23). Moreover, the Outpatients Clinic is staffed with skilled professionals, and extensive experience in breast/ ovarian patients follow-up was established and was specifically dedicated to the present study. This set-up allowed all patients treated for primary surgery in the Women's Department of NCRC, Bari to be enrolled in the present study.

The aim of the study was to verify the mutation spectra and clinical outcome of patients and relatives with familial features carrying or not carrying BRCA mutations in a consecutive monoinstitutional and sufficiently large series of patients from Southern Italy. Data from a median follow-up of 78 months was available.

\section{Materials and methods}

Subjects. Between June, 2003 and June, 2010, a series of $\sim 5,000$ women with breast cancer were consecutively treated for a first diagnosis of breast cancer at the Breast Unit of the Women's Department of NCRC, Bari, Italy. Among these, all patients with familial characteristics (at least one first-degree relative) were referred to the Genetic Counseling Outpatient Clinic of the Institute for further data collection. After a family tree reconstruction and calculation of the BRCA mutation probability according to BRCAPRO, 136 women with breast cancer and a BRCAPRO risk $>10 \%$ were considered to be candidates and underwent a BRCA mutation test. Among these, 79 patients in the period 2003-2010 and 57 patients before 2003 were identified and underwent surgical resection for primary breast cancer. All women who underwent genetic testing for $B R C A 1$ and $B R C A 2$ mutations and provided informed consent for participation in this study were considered for inclusion.

The median follow-up time for 132 available affected patients was 6.5 years (range, 1-42) with a total of 1,195 personyears of follow-up.

Furthermore, during the same period, 66 relatives from 45 families underwent BRCA mutation testing if belonging to BRCA mutation carrier families. Recruitment of living parents and adult siblings of the probands was carried out wherever possible. Documented verification of reported cancers (through pathology reports, cancer registries and medical records) was also carried out.

For each patient and relative involved in this study the following information was available: i) exact mutation present in the family, ii) date of genetic testing, iii) age at development of the cancer, iv) cancer type, v) surgical, radiation therapy and systemic adjuvant treatment, vi) pathological information such as tumor size, lymph node status, histological type, estrogen (ER) and progesterone receptors (PgR), histological grade, proliferation index and HER2 receptor expression, vii) followup information on the following endpoints: ipsilateral breast tumor recurrence (IBTR), CBC, distant metastasis (M), disease-free survival and new cases of cancer.

BRCA1 and BRCA2 mutation testing. The genomic DNA samples were screened for mutations in the $B R C A 1$ and $B R C A 2$ genes by a sequential combination of denaturing high performance liquid chromatography (DHPLC) analysis and direct sequencing (AB 3130xl). All relatives were screened only for the mutation detected in the index case of the family.

Statistical analysis. Disease-free survival (DFS) was defined as the time from cancer diagnosis to documented evidence of disease recurrence in locoregional and/or distant sites, the manifestation of a CBC, a second primary cancer in a nonbreast site. The DFS probability [95\% confidence interval (CI)] was computed using the Kaplan-Meier product-limit method. The null hypothesis concerning the differential effect of carrier status in the univariate analysis was tested by logrank test (24).

Estimated hazard ratios (the ratio of the carrier group to the control group) were calculated from proportional hazard regression models stratified according to lymph node status, tumor size and surgery. The multivariate model was used to investigate potential confounding factors (25). For unaffected subjects at the time of genetic testing, the age-specific cumulative cancer incidence was estimated by Kaplan-Meier survival functions in which failure time was age at cancer diagnosis. All statistical analyses were carried out using SPSS statistical software (26).

\section{Results}

Among the 136 probands analyzed for the molecular status of the BRCA1/BRCA2 genes, 49 cases had a mutation in the BRCA1 $(n=33)$ or BRCA2 $(n=17)$ gene, with 1 case carrying a mutation in both genes; conversely, 87 women did not show any mutations in either BRCA1 or the two genes. The spectrum of mutations in both genes is documented in Fig. 1.

Most mutations censored in BRCA1 were c.5266dupC and c. $181 \mathrm{~T}>\mathrm{G}$, while in BRCA2 they were c.6462_6463delTC, c.5796_5797delTA and c.1772_1776delTTTA.

In Table I, all first disease-related events at a median follow-up of 6.5 years are reported. A total of 44/136 (33.3\%) women presenting with a disease-related event were noted: $4(2.9 \%)$ patients had a relapse in the ipsilateral breast and $5(3.7 \%)$ in locoregional areas; $18(13.2 \%)$ women presented with a new cancer site in the contralateral breast; $12(8.8 \%)$ presented metastases in distant sites; finally, 4 (2.9\%) women had a second primary cancer in the ovaries and $1(0.7 \%)$ case had a multiple myeloma.

When the disease-related events were analyzed with respect to the BRCA1/2 status, the probability of recurrence was slightly higher ( $\mathrm{P}>0.05$ by Chi-square test) in mutation carriers $(20 / 49 ; 40.8 \%)$ than in non-carriers $(24 / 87 ; 27.5 \%)$. 
A

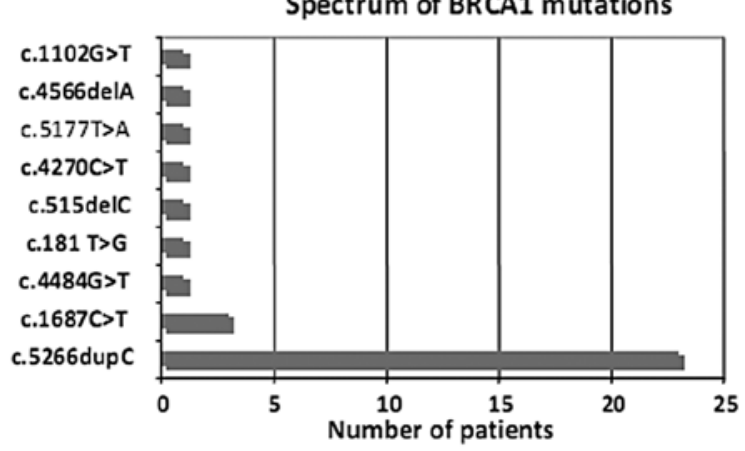

B Spectrum of BRCA2 mutations

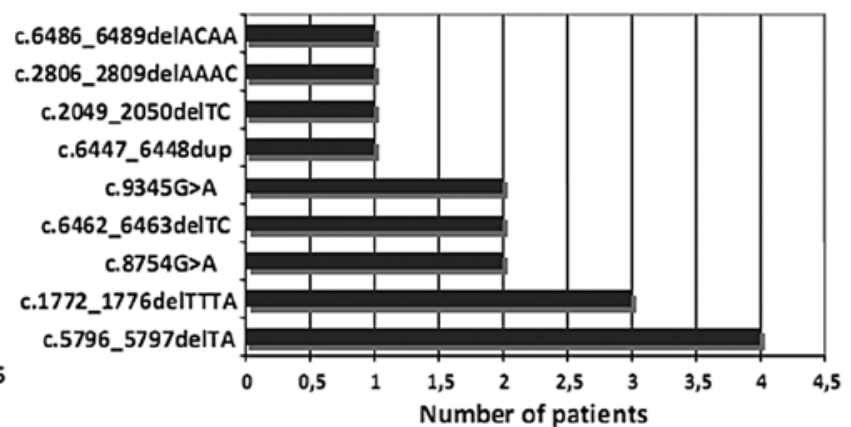

Figure 1. Spectrum of BRCA1/2 mutations censored in 49 patients with breast cancer. (A) Patients carrying the BRCA1 gene mutation (n=32). (B) Patients carrying the BRCA2 gene mutation $(\mathrm{n}=17)$. One woman presented with a mutation in both genes (BRCA1, c.5266dupC; BRCA2, c.5796_5797delTA).

Table I. First event profile in probands at follow-up of 6.5 years.

\begin{tabular}{lcccrr}
\hline & \multicolumn{3}{c}{ Molecular status C=49 } & \\
\cline { 2 - 5 } Site of disease & BRCA1 (32) & BRCA2 (16) & BRCA1/2 (1) & NC=87 & Overall \\
\hline Ipsilateral & $1(3 \%)$ & 0 & 1 & 2 & 4 \\
Contralateral & $6(19 \%)$ & 1 & - & 11 & 18 \\
Locoregional & 2 & 1 & - & 2 & 5 \\
Distant metastasis & $2^{\mathrm{a}}$ & $2^{\mathrm{b}}$ & - & $8^{\mathrm{c}}$ & 12 \\
Ovarian & 3 & 0 & 1 & 0 & 4 \\
Other & $1^{\mathrm{d}}$ & $4 / 16(25 \%)$ & - & - \\
\cline { 2 - 6 } Total patients with relapse & $15 / 32(46.8 \%)$ & 20 & 24 & 44 \\
\hline
\end{tabular}

${ }^{\mathrm{a}} 1$ bone, 1 lung; ${ }^{\mathrm{b}} 1$ lung, 1 bone + liver; ${ }^{\mathrm{c}} 3$ bone, 2 lung, 1 lung + liver, 1 bone + liver, 1 suvraclavear; ${ }^{\mathrm{d}}$ multiple myeloma. C, carrier; $\mathrm{NC}$, non-carrier.

Table II. Overall ipsilateral and contralateral breast cancer relapses.

\begin{tabular}{lccccc}
\hline & \multicolumn{3}{c}{ Molecular status C=49 } \\
Site of disease & BRCA1 (32) & BRCA2 (16) & BRCA1/2 (1) & NC=87 & $\chi^{2}$ \\
\hline Ipsilateral & 3 & - & 1 & 6 & $\mathrm{P}=0.785$ \\
Contralateral & $8(25 \%)$ & $1(6 \%)$ & - & 13 & $\mathrm{P}=0.848$ \\
\hline
\end{tabular}

$\mathrm{C}$, carrier; NC, non-carrier.

Notably, the probability reached $47 \%$ (15/32) when considering only the BRCA1 mutation.

Regarding analysis of all CBCs observed during the follow-up period, a total of 9 out of 49 carriers and 13 out of 87 non-carrier events were observed ( $\mathrm{P}=0.848$, Chi-square test) (Table II). Notably, CBC principally occurred as a first event ( $80 \%$ of all contralateral relapses).

In particular, 8 patients with BRCA1 mutations $(5$ cases with c.5266dupC, 1 case with c. $1687 \mathrm{C}>\mathrm{T}$, 1 case with c.515delC and 1 case with c.5177T $>$ A), and 1 patient with a BRCA2 mutation (c.6486_6489delACAA) presented with CBCs. Ovarian cancers were observed in 3 cases with the BRCA1 mutation ( 2 cases with c.5266dupC and 1 case with c.1687C $>$ T).

Regarding DFS curves, the 10-year DFS was $57 \%$ for the subgroup of women not carrying a mutation with respect to $50 \%$ in mutation carriers (Fig. 2) ( $\mathrm{P}=0.1529$ by log-rank test). In order to investigate whether other clinicopathological confounding variables could mask the clinical impact of a genetic alteration on clinical outcome, a multivariate analysis was performed with DFS as a dependent variable and genetic status, tumor size, nodal status and type of primary surgery included in the model. 
Table III. Cox's proportional hazard regression models for disease-free survival.

\begin{tabular}{|c|c|c|c|c|}
\hline & & HR & P-value & $(95 \% \mathrm{CI})$ \\
\hline Variable & $\mathrm{n}(\%)$ & & & \\
\hline All cases & $112(82)$ & & & \\
\hline BRCA $^{-}$vs. BRCA $^{+}$ & & 1.49 & 0.240 & $0.76-2.91$ \\
\hline T1 vs. T2-T4 & & 0.27 & 0.049 & $0.07-0.99$ \\
\hline $\mathrm{N}^{-}$vs. $\mathrm{N}^{+}$ & & 2.61 & 0.126 & $0.76-8.96$ \\
\hline M vs. BCS & & 0.55 & 0.112 & $0.27-1.14$ \\
\hline
\end{tabular}

HR, hazard ratio; CI, confidence interval; N, lymph node status; $\mathrm{M}$, mastectomy; BCS, breast-conserving surgery.

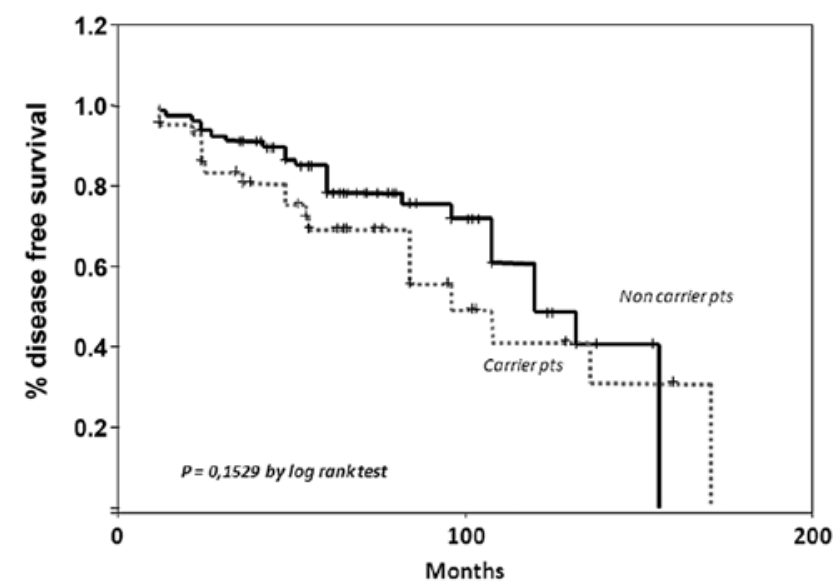

Figure 2. Disease free survival (DFS) of patients with respect to first event in BRCA carrier $(n=49)$ and non-carrier $(n=87)$ groups with breast cancer.

The Cox analysis demonstrated that the time of relapse was significantly associated (HR 0.27; P<0.49; CI, 0.07-0.99) solely with tumor size (Table III).

When overall survival was analyzed, we observed 15 deaths; 7 in the mutation carrier group and 8 in the group without mutations, with a 10 -year overall survival of 81 vs. 92\% ( $\mathrm{P}=0.6091$ by log-rank test).

Finally, we examined outcome in the group of 66 healthy relatives carrying $(n=32)$ or not carrying $(n=34)$ a BRCA gene mutation. In these subgroups, 6 new cancers ( 5 cases of breast cancer and 1 case of ovarian cancer) were observed in women carrying a mutation vs. no new cases in the healthy relatives not carrying a gene mutation $(\mathrm{P}=0.02$, Chi-square test) (Table IV). Notably, the median age of the two subgroups was not significantly different at the time of the last followup (median age, 40.5 vs. 41 , for carriers and control groups, respectively) with a $40 \%$ risk of breast/ovarian cancer at the age of 50 years in women carrying a BRCA mutation (Fig. 3) ( $\mathrm{P}=0.0069$ by log-rank test).

\section{Discussion}

Our study allowed us to obtain information on two main aspects concerning the clinical-biological role of the BRCA mutation: i) to compare the clinical outcome of breast cancer
Table IV. New cancer diagnoses in relatives unaffected at the time of genetic testing $(\mathrm{n}=66)$.

\section{Molecular status}

Site of disease BRCA1 (13) BRCA2 (19) $\mathrm{C}=32 \quad \mathrm{NC}=34$

\begin{tabular}{lllll}
\hline Breast cancer & 3 & 2 & 5 & 0 \\
Ovarian cancer & 1 & - & 1 & 0 \\
Total & & & 6 & 0
\end{tabular}

C, carrier; NC, non-carrier.

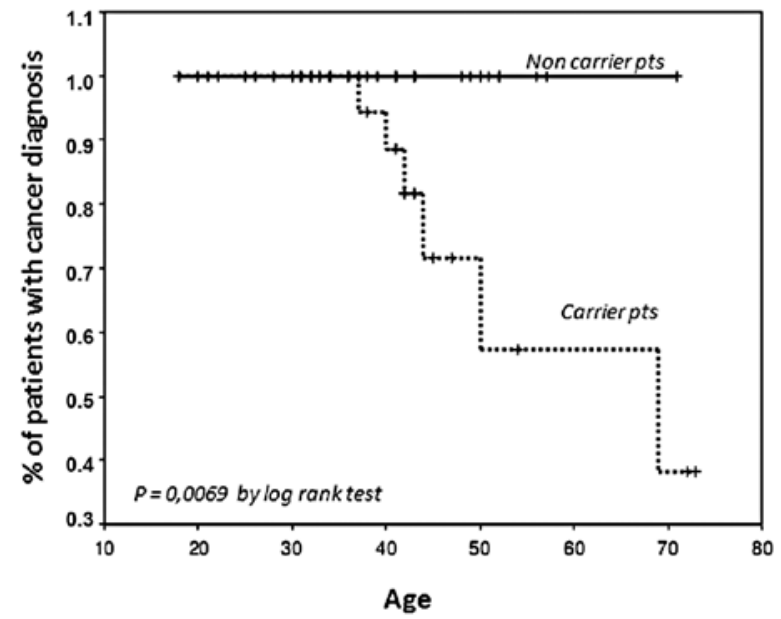

Figure 3. New cases of cancer in unaffected relatives at the time of genetic testing carrying $(n=32)$ or not carrying $(n=34)$ BRCA mutations.

women carrying or not carrying a BRCA mutation; ii) the risk for breast/ovarian cancer in healthy relatives carrying or not carrying a BRCA mutation.

The unique characteristics of our study must be stressed. We enrolled a monoinstitutional consecutive series of patients, molecularly characterized at one laboratory who received primary surgery/radiotherapy/systemic treatment/follow-up at the same institute and according to standardized guidelines.

We demonstrated that for patients belonging to a hereditary breast cancer group, the presence or not of a BRCA mutation does not alter the clinical outcome of women with breast cancer. In fact, the probability of a relapse is higher but not statistically significant in carriers of a mutation with respect to those with no mutation.

The lack of statistical power for this comparison could be supported by the absence of a difference between the two groups, but also the possibility that the study concerned consisted of a limited number of women. A larger series of women should be studied using the same methodology as applied in the present study.

The evidence of the absence of a relationship with clinical outcome is supported by several previous studies, including that of Rennert et al (13) who analyzed the largest series ever considered $(n=1794)$. However, our analysis referred to familial high-risk women eventually presupposing the presence of a genetic alteration other than BRCA1/2 mutations. 
The lack of difference also concerns the contralateral appearance of the disease. This is in contrast with the results of Robson et al (27), Kirova et al (28) and Brekelmans et al (29) who demonstrated a higher risk for contralateral disease in BRCA carriers with respect to non-carriers.

It has been shown that age remains a significant predictor of ipsilateral breast cancer recurrence and, in particular, that young age, more than the BRCA status, is a strong predictive factor for local relapse among hereditary breast cancer patients $(28,30)$.

Thus, to ascertain how young age is important in CBC, following adjustment for age, we detected all (9/9) contralateral recurrences out of 49 in mutation carriers compared with $70 \%(10 / 13)$ of cases out of 89 in non-carriers in a population with a diagnosis of first breast cancer at an age ranging from 20 to 49 years $(\mathrm{P}=0.394$, Chi-square test). Due to the small number of patients, this difference was not significant.

However, a more extended follow-up is needed to ensure that the rate of $\mathrm{CBC}$ in young age $\mathrm{BRCA}^{+}$patients does not increase in the long-term.

Finally, we investigated the risk of breast cancer in healthy relatives carrying or not carrying a BRCA gene mutation. Notably, in this further analysis, we demonstrated that the risk for breast and ovarian cancers in relatives was significantly different in carriers with respect to non-carriers, with a $43 \%$ risk at an age of 50 years in women carrying a BRCA mutation.

In conclusion, our data indicate that in women with a deleterious mutation in BRCA1 or BRCA2, clinical outcome is no worse than in BRCA-negative patients, while it results in a significant difference from the outcome of sporadic breast cancer patients. Therefore, we are currently recruiting a large series of sporadic breast cancer patients in order to compare the results with the present findings. Identification of the BRCA status in relatives of breast cancer patients carrying the mutation is crucial for the prediction of risk.

\section{Acknowledgements}

The present study was supported, in part, by Progetto Regione Puglia ‘Familial Breast Cancer Screening' (DIEF 2007).

\section{References}

1. Emery J, Lucassen A and Murphy M: Common hereditary cancers and implications for primary care. Lancet 358: 56-63, 2001.

2. Brose MS, Rebbeck TR, Calzone KA, et al: Cancer risk estimates for BRCA1 mutation carriers identified in a risk evaluation program. J Natl Cancer Inst 94: 1365-1372, 2002.

3. Easton DF, Bishop DT, Ford D and Crockford GP: Genetic linkage analysis in familial breast and ovarian cancer: results from 214 families. The Breast Cancer Linkage Consortium. Am J Hum Genet 52: 678-701, 1993.

4. King MC, Marks JH and Mandell JB; New York Breast Cancer Study Group: Breast and ovarian cancer risks due to inherited mutations in BRCA1 and BRCA2. Science 302: 643-646, 2003.

5. Antoniou A, Pharoah PD, Narod S, et al: Average risks of breast and ovarian cancer associated with BRCA1 or BRCA2 mutations detected in case series unselected for family history: a combined analysis of 22 studies. Am J Hum Genet 72: 1117-1130, 2003.

6. Cancer risks in BRCA2 mutation carriers. The Breast Cancer Linkage Consortium. J Natl Cancer Inst 91: 1310-1316, 1999.

7. Liede A, Karlan BY and Narod SA: Cancer risks for male carriers of germline mutations in BRCA1 or BRCA2: a review of the literature. J Clin Oncol 22: 735-742, 2004.
8. Metcalfe K, Lubinski J, Lynch HT, et al: Family history of cancer and cancer risk in women with BRCA1 or BRCA 2 mutations. J Natl Cancer Inst 102: 1874-1878, 2010.

9. Lee EH, Park SK, Park B, et al: Effect of BRCA1/2 mutation on short-term and long-term breast cancer survival: a systematic review and meta-analysis. Breast Cancer Res Treat 122: 11-25, 2010.

10. Adem C, Reynolds C, Soderberg CL, et al: Pathologic characteristics of breast parenchyma in patients with hereditary breast carcinoma, including BRCA1 and BRCA2 mutation carriers. Cancer 97: 1-11, 2003.

11. Chappuis PO, Nethercot V and Foules WD: Clinico-pathological characteristics of BRCA1 and BRCA2-related breast cancer. Semin Surg Oncol 18: 287-295, 2000.

12. Pathology of familial breast cancer: differences between breast cancers in carriers of BRCA1 or BRCA2 mutations and sporadic cases. The Breast Cancer Linkage Consortium. Lancet 349: 1505-1510, 1997.

13. Rennert G, Bisland-Naggan S, Barnett-Griness O, et al: Clinical outcomes of breast cancer in carriers of BRCA1 and BRCA2 mutations. N Engl J Med 357: 115-123, 2007.

14. Budroni M, Cesaraccio R, Coviello V, et al: Role of BRCA2 mutation status on overall survival among breast cancer patients from Sardinia. BMC Cancer 9: 62, 2009.

15. Verhoog LC, Brekelmans CT, Seynaejjve, et al: Contralateral breast cancer risk is influenced by the age at onset in BRCA1associated breast cancer. Br J Cancer 83: 384-386, 2000.

16. Metcalfe K, Lynch HT, Ghadirian P, et al: Contralateral breast cancer in BRCA1 and BRCA2 mutation carriers. J Clin Oncol 22: 2328-2335, 2004.

17. Pierce LJ, Levin AM, Rebbeck TR, et al: Ten-year multi-institutional results of breast-conserving surgery and radiotherapy in BRCA1/2-associated stage I/II breast cancer. J Clin Oncol 24: 2437-2443, 2006

18. Sariego J: The impact of facility volume/size on breast cancer treatment and outcome. Am Surg 76: 1333-1337, 2010.

19. Paradiso A, Giardina C and Meyer JS: Statewide study of diagnostic agreement in breast pathology. J Natl Cancer Inst 91: 1076-1077, 1999.

20. Early Breast Cancer Trialists' Collaborative Group (EBCTCG); Peto R, Davies C, Godwin J, et al: Comparisons between different polychemotherapy regimens for early breast cancer: meta-analyses of long-term outcome among 100,000 women in 123 randomised trials. Lancet 379: 432-444, 2012.

21. Tommasi S, Crapolicchio A, Lacalamita R, et al: BRCA1 mutations and polymorphisms in a hospital-based consecutive series of breast cancer patients from Apulia, Italy. Mutat Res 578: 395-405, 2005

22. Tommasi S, Pilato B, Pinto R, et al: Molecular and in silico analysis of BRCA1 and BRCA2 variants. Mutat Res 644: 64-70, 2008.

23. Mueller CR, Kristoffersson U and Stoppa-Lyonnet D: External quality assessment for mutation detection in the BRCA1 and BRCA2 genes: EMQN's experience of 3 years. Ann Oncol 15 (Suppl 1): i14-i17, 2004.

24. Lawless JS: Statistical Models and Methods for Lifetime Data. John Wiley and Sons, New York, NY, 1982

25. Cox DR: Regression models and life tables. JR Stat Soc 34: 187-220, 1972 .

26. Sweet SA: Data Analysis with SPSS. Allyn and Bacon, Boston, MA, 1998.

27. Robson M, Chappuis PO, Satagopan J, et al: A combined analysis of outcome following breast cancer: differences in survival based on BRCA1/BRCA2 mutation status and administration of adjuvant treatment. Breast Cancer Res 6: R8-R17, 2004.

28. Kirova YM, Stoppa-Lyonnet D, Savignoni A, et al: Risk of breast cancer recurrence and contralateral breast cancer in relation to BRCA1 and BRCA2 mutation status following breast-conserving surgery and radiotherapy. Eur J Cancer 41: 2304-2311, 2005.

29. Brekelmans CTM, Tilanus-Linthorst MMA, Seynaeve C, et al: Tumor characteristics, survival and prognostic factors of hereditary breast cancer from BRCA2-, BRCA1- and non-BRCA1/2 families as compared to sporadic breast cancer cases. Eur J Cancer 43: 867-876, 2007.

30. Delaloge S, Kloos I, Ariane D, et al: Young age is the major predictor of local relapse among conservatively treated BRCA1-, BRCA2-, or non BRCA-linked hereditary breast cancer (BC). Proc Am Soc Clin Oncol 22: 41, 2003. 